\title{
Cabazitaxel liposomes with aptamer modification enhance tumor-targeting efficacy in nude mice
}

\author{
YUZHU CHENG $^{1 *}$, ZHANLUN OU $^{1 *}$, QINGGUO LI ${ }^{1 *}$, JUAN YANG $^{1}$, MIN HU $^{1}$, YUBIN ZHOU $^{2}$, \\ XIAODONG ZHUANG ${ }^{3}$, ZHENYU JASON ZHANG ${ }^{4}$ and SHIXIA GUAN ${ }^{1}$ \\ ${ }^{1}$ Department of Pharmacy, School of Pharmaceutical Sciences, Guangzhou University of Chinese Medicine, \\ Guangzhou, Guangdong 510006, P.R. China; ${ }^{2}$ School of Materials Science and Engineering, \\ College of Engineering, Nanyang Technological University, Singapore 639798, Republic of Singapore; \\ ${ }^{3}$ Target Discovery Institute NDM Research Building, University of Oxford, Oxford OX3 7FZ; \\ ${ }^{4}$ School of Chemical Engineering, University of Birmingham, Birmingham B15 2TT, UK
}

Received June 20,2018; Accepted October 25, 2018

DOI: $10.3892 / \mathrm{mmr} .2018 .9689$

\begin{abstract}
The present study investigated the feasibility of improving the tumor-targeting efficacy and decreasing the toxicity of liposomal cabazitaxel (Cab) with aptamer modification. The process involved preparing aptamer (TLS1c)-modified liposomes and studying the behavior of the liposomes in vitro and in vivo. TLS1c as an aptamer, which has high specificity for BNL 1ME A.7R.1 (MEAR) cells, was conjugated with Cab liposomes (Cab/lipo) to enhance MEAR tumor tissue targeting. Confocal laser scanning microscopy and flow cytometry analyses demonstrated that the fluorescence of the liposomes modified with the aptamer was notably stronger compared with that of the unmodified liposomes. Furthermore, the biodistribution data of the modified liposomes tested in tumor-bearing mice revealed high specificity of biotinylated TLS1c-modified Cab/lipo (BioTL-Cab/lipo) for tumor tissues. Furthermore, the modified liposomes demonstrated decreased cytotoxicity and simultaneously retained potent inhibition against tumor growth. It is likely that the specific binding of the aptamer (TLS1c) to the targeted cells (MEAR) facilitates the binding of the liposomes to the targeted cells. Therefore, BioTL-Cab/lipo may be considered as a promising delivery system to improve cell targeting and reduce drug toxicity in the treatment of cancer.
\end{abstract}

Correspondence to: Dr Shixia Guan, Department of Pharmacy, School of Pharmaceutical Sciences, Guangzhou University of Chinese Medicine, 232 University City Ring Road East, Panyu, Guangzhou, Guangdong 510006, P.R. China

E-mail:drguan@gzucm.edu.cn

${ }^{*}$ Contributed equally

Key words: aptamers, cabazitaxel, liposomes, tumor-targeting, TLS1c, nude mice

\section{Introduction}

Cancer remains a problem worldwide and the majority of patients suffer from the severe side effects of anticancer drugs (1). There is an urgent requirement to improve the specificity of cancer drugs to tumors. At present, aptamers, which are typically small single-stranded DNA/RNA oligonucleotides or small peptides generated by artificial systemic evolution of ligands by exponential enrichment (2), have been demonstrated to bind to a wide variety of targets, including small molecules, proteins, cells and tissues, with high affinity and selectivity (3-6). Owing to their various advantages, aptamers have been used in biomarker identification, disease diagnosis, therapies, and most promising, as targeting elements for developing novel drug delivery systems $(7,8)$. Cabazitaxel (Cab) is an antineoplastic agent with a broad antitumor spectrum; however, its use in the clinical setting has been hindered by certain adverse effects. (9) Novel targeting biotin-conjugated TLS1c Cab liposomes (BioTL-Cab/lipo) have been designed to investigate whether they may decrease the cytotoxicity of $\mathrm{Cab}$ in mice while retaining its antitumor properties. Increasing efforts have been made in the field of aptamer-based tumor-targeting; however, few studies have demonstrated the effects and characteristics of aptamers in Cab-associated liposomes.

Shangguan et al (10) observed that TLS1c, an aptamer with a short DNA sequence, specifically targeted MEAR hepatoma cells. The synthetic nucleic acid nature of TLS1c enables easy and simple conjugation with biotin, enhancing its stability in biological fluids (11). The biotinylated TLS1c (BioTL) was selected as a targeting recognition element for MEAR hepatoma in the present study. Liposomes, as a traditional passive targeting system (12), have been widely investigated in drug administration. Liposomes may decrease the cytotoxicity of drugs; however, to the best of our knowledge, $\mathrm{Cab}$ liposomes (Cab/lipo) have not been previously investigated.

In the present study,DNA aptamerTLS1c-modified Cab/lipo was used to investigate tumor-targeting efficiency, where the direct comparison between Cab/lipo and BioTL-Cab/lipo was 
reported for the first time, to the best of our knowledge. The cytotoxicity of the prepared liposomes was investigated in Caco-2, HepG2 and MEAR cell lines, and it was identified that BioTL-Cab/lipo exhibited higher cytotoxicity in MEAR cells compared with Caco-2 and HepG2 cells. Consistent results were obtained with confocal laser scanning microscopy (CLSM) and flow cytometry analysis; highly specific targeting of the prepared BioTL-Cab/lipo to MEAR cells was demonstrated. Furthermore, the biodistribution and in vivo antitumor efficacy were monitored in MEAR tumor-bearing animal models. The accumulation of BioTL-Cab/lipo in tumor tissue was demonstrated with real-time images. Collectively, the results of the present study suggested that TLS1c-modified Cab/lipo demonstrated a specific and potent antitumor effect in vivo.

\section{Materials and methods}

Materials. Soya phosphatidyl choline (LIPOID S 100; SPC) was purchased from Lipoid GmbH (Ludwigshafen, Germany). Ethyl polyenoate (EP) was obtained from Shandong Yuwang Pharmaceutical Co., Ltd. (Yucheng, China). Cab was supplied by Guangzhou Zhiyuan Pharmacy Co., Ltd. (Guangzhou, China). Sodium cholesterol sulfate (SCS) was purchased from Shanghai Haoran Biotechnology Co., Ltd. (Shanghai, China). BioTL was purchased from Genewiz, Inc. (Beijing, China). Tris (hydroxymethyl) methyl aminomethane (Tris) was obtained from Jian Yang Biotechnology Co., Ltd. (Guangzhou, China). The nucleotide sequence of TLS1c is 5'-ACAGGAGTGATGGTTGTT ATCTGGCCTCAGAGGTTCTCGGGTGTGGTCACTCCT G-3'. TLS1c was purchased from Genewiz, Inc.; biotin was conjugated with TLS1c at the 3' end. Shanghai Macklin Biochemical Co., Ltd. (Shanghai, China) provided 6-carboxy fluorescein (6-FAM). Harvey Biotech Co., Ltd. (Beijing, China) provided 1,1'-dioctadecyl-3,3,3',3'-tetramethylindocarbocyanine perchlorate (DiI). HepG2, macrophages and MEAR cells were provided by Shanghai Fuxiang Biotechnology Co., Ltd. (Shanghai, China). Caco-2 cells were obtained from the American Type Culture Collection (Manassas, VA, USA). The TDL-40B Ultrafiltration centrifuge was supplied by Shanghai Anting Science Technology Instrument Factory (Shanghai, China). All other chemical and buffer components were of analytical grade.

Preparation of liposomes. Liposomes were prepared by reverse-phase evaporation of vesicles (13). The organic phase (OP) comprised $8 \mathrm{mg} \mathrm{Cab}, 120 \mathrm{mg}$ SPC, $12 \mathrm{mg}$ SCS and $16 \mathrm{mg}$ EP (a surfactant) dissolved in $3 \mathrm{ml}$ dichloromethane. The aqueous phase (AP) constituted $20 \mathrm{ml}$ Tris- $\mathrm{HCl}(\mathrm{pH} 7.5)$. The OP was deposited into the AP with stirring (600 rpm) for $45 \mathrm{~min}$ at $25^{\circ} \mathrm{C}$. The emulsion was transferred to a container and BioTL in Tris- $\mathrm{HCl}$ was added. Probe sonication (UH-500A ultrasonic processor; Tianjin Automatic Science Instruments Co., Ltd., Tianjin, China) was conducted for $15 \mathrm{~min}$ in an ice-water bath. Dichloromethane was evaporated using a Rotary Evaporator (RE-2000; Shanghai Yarong Bio, Inc., Shanghai, China) at $45^{\circ} \mathrm{C}$, for $15 \mathrm{~min}$ and $100 \mathrm{r} / \mathrm{min}$. The BioTL-Cab/lipo was freeze-dried using a Tofflon LY0-0.5 freeze dryer (Shanghai Tofflon Technology
Co., Ltd., Shanghai, China) with 5\% (w/v) lactose solution as the freeze-drying protective additive. Non-BioTL-containing liposomes (Cab/lipo) were prepared using the same composition and method as BioTL-Cab/lipo without the addition of BioTL solution. To examine the behavior of liposomes in vivo and in vitro, Cab was replaced with 6-FAM (the content was $2 \mathrm{mg}$ 6-FAM/lipo or BioTL-6-FAM/lipo) by the same procedure.

Physicochemical characterization of liposomes. The particle size polydispersity index, and the $\zeta$ potential were determined using a Zetasizer Nano ZS analyzer (Malvern Instruments, Ltd., Malvern, UK). The content of the loaded Cab was measured by high performance liquid chromatography (HPLC) analysis (LC-10A, Shimadzu Corporation, Kyoto, Japan) equipped with an ODS C18 chromatographic column (4.6x250 mm; $5 \mu \mathrm{m}$; Thermo Fisher Scientific, Inc., Waltham, MA, USA). The mobile phase was composed of methanol, acetonitrile and water $(35: 40: 25 ; \mathrm{v} / \mathrm{v})$. A constant flow rate of $1 \mathrm{ml} / \mathrm{min}$ and detection wavelength of $240 \mathrm{~nm}$ were applied for analysis at room temperature. The content was calculated using a linear calibration curve of standard Cab solutions in $\mathrm{MeOH}$.

The encapsulation efficiency (EE) of Cab or 6-FAM was calculated as a percentage of the dissociated $\mathrm{Cab}$ from the suspension using 3,500 rpm for $5 \mathrm{~min}$ in order for the total Cab content of the liposomes to be degraded by methanol in the buffer; the nominal molecular weight limit of the ultrafiltration membrane was $3 \mathrm{kDa}$. The following formula was used to calculate the $\mathrm{EE} \%$ of liposomes:

$$
\mathrm{EE} \%=\frac{(\text { Total content }- \text { Dissociate } \mathrm{d} \text { content })}{\text { Total content }} \times 100
$$

The effects of the holding capacity of BioTL on the EE\% of liposomes was validated using an Ultra-Micro ultraviolet (UV) spectrophotometer (Thermo Fisher Scientific, Inc.). Due to the nucleic acid characterization of UV absorption, the content of BioTL was determined by absorption at $260 \mathrm{~nm}$.

Additionally, the optimal ratio of aptamers to SPC $(\mu \mathrm{g} / \mathrm{mg})$ was determined using a flow cytometer (BD FACSCanto ${ }^{\mathrm{TM}} \mathrm{II}$; BD Biosciences, Franklin Lakes, NJ, USA). MEAR cells were cultured at $37^{\circ} \mathrm{C}$ in $5 \% \mathrm{CO}_{2}$ and $95 \%$ humidity in Dulbecco's modified Eagle's medium (Gibco; Thermo Fisher Scientific, Inc.) supplemented with $10 \%$ fetal bovine serum (Gibco; Thermo Fisher Scientific, Inc.). MEAR cells were counted and seeded on 6 -well plates $\left(1 \times 10^{6}\right.$ cells/well), and cultured overnight at $37^{\circ} \mathrm{C}$ in the incubator. Subsequently, cells were treated with different BioTL-6-FAM/lipo to SPC rates $(0.2$, 1 and $2 \mu \mathrm{g} / \mathrm{mg}$ ) for 1,3 and $5 \mathrm{~h}$ at $37^{\circ} \mathrm{C}$ to determine the best ratio. The concentration of BioTL was $1.2,6,12 \mu \mathrm{g} / \mathrm{ml}$, respectively, and the concentration of SPC used as the control was $6 \mathrm{mg} / \mathrm{ml}$.

The medium was removed at the aforementioned time points; cells were washed with $\mathrm{PBS}$ and subsequently harvested using $0.05 \%$ trypsin at $37^{\circ} \mathrm{C}$ for $1 \mathrm{~min}$. The fluorescence intensity of 6-FAM in cells was determined with excitation and emission wavelengths of 452 and $521 \mathrm{~nm}$ respectively using the flow cytometer. 
Cytotoxicity assays. HepG2, macrophages and MEAR cells were cultured at $37^{\circ} \mathrm{C}$ in $5 \% \mathrm{CO}_{2}$ and $95 \%$ humidity in Dulbecco's modified Eagle's medium (Gibco; Thermo Fisher Scientific, Inc.) supplemented with $10 \%$ fetal bovine serum (Gibco; Thermo Fisher Scientific, Inc.), Caco-2 cells were cultured under the same conditions with an additional $1 \%$ non-essential amino acids. The cytotoxic effect of the Cab solution was initially assessed using MEAR cells ( $5 \times 10^{3}$ cells/well), which were seeded in 96-well plates (Corning Incorporated, Corning, NY, USA), and cultured overnight. Cells were incubated with fresh medium containing different concentrations of $\mathrm{Cab}(0.0625,0.125,0.25,0.5,1$ and $2 \mu \mathrm{g} / \mathrm{ml})$. After 24,48 or $72 \mathrm{~h}$ incubation, MTT $(5 \mathrm{mg} / \mathrm{ml} ; 20 \mu \mathrm{l})$ was added to each well for a further incubation of $4 \mathrm{~h}$ at $37^{\circ} \mathrm{C}$. Subsequently, the medium was removed and $100 \mu \mathrm{l}$ dimethyl sulfoxide (DMSO) was added to dissolve the formazan crystals with $10 \mathrm{~min}$ agitation. The optical density was determined at $490 \mathrm{~nm}$ using a spectrophotometer (Multiskan GO, Thermo Fisher Scientific, Inc.). The cytotoxicity of the Cab/lipo and BioTL-Cab/lipo was evaluated using Caco-2, HepG2 and MEAR cells. BioTL-Cab/lipo $(0.5 \mu \mathrm{g} / \mathrm{ml}), \mathrm{Cab} / \mathrm{lipo}(0.5 \mu \mathrm{g} / \mathrm{ml})$ and $\mathrm{Cab}$ dissolved in Tris- $\mathrm{HCl}$ $(0.5 \mu \mathrm{g} / \mathrm{ml})$ were incubated with cells $24 \mathrm{~h}$ later at $37^{\circ} \mathrm{C}$. The optical density was determined as described above.

Cellular uptake studies. Caco-2, HepG2 and MEAR cells were seeded in a 12 -well culture plate $\left(2 \times 10^{5}\right.$ cells/well). After $24 \mathrm{~h}$, cells were incubated with fresh medium containing 6-FAM/lipo or BioTL-6-FAM/lipo at $37^{\circ} \mathrm{C}$ for $3 \mathrm{~h}$. The final concentration of $6-\mathrm{FAM}$ was $6 \mu \mathrm{g} / \mathrm{ml}$. After $3 \mathrm{~h}$ incubation, cells were washed with cold PBS three times. Cells were stained with DAPI (Biosharp, Nanjing, China) at room temperature for $20 \mathrm{~min}$. The cells were washed three times with PBS under agitation. Cells were fixed on slides with cold acetone $\left(-20^{\circ} \mathrm{C}\right)$ at room temperature and visualized via CLSM (Carl Zeiss AG, Oberkochen, Germany) at x400 magnification, images were acquired of $\geq 5$ fields per view.

Caco-2, HepG2 and MEAR cells (1x10 6 cells/well) were seeded in the 6 -well plates and cultured overnight. Cells were incubated with fresh medium containing 6-FAM/lipo, BioTL-6-FAM/lipo or Tris- $\mathrm{HCl}$ at $37^{\circ} \mathrm{C}$ for $3 \mathrm{~h}$. The cells treated with Tris- $\mathrm{HCl}$ served as the control group. Flow cytometry was conducted as described above.

Biodistribution in mice. Male BALB/c nude mice $(\mathrm{n}=6$; 5 weeks-old, $\sim 20 \mathrm{~g}$ ) were housed in a temperature-controlled room $\left(22-25^{\circ} \mathrm{C}\right)$, with $50-60 \%$ relative humidity under a $12 \mathrm{~h}$ light-dark cycle with free access to food and water. All animal treatments were performed in accordance with the Regulations of the Administration of Affairs Concerning Experimental Animals (http://en.pkulaw.cn/display.aspx?cgid =3f3dc921e8e837aebdfb\&lib=law) and were approved by the Animal Research Ethics Committee at Guangzhou University of Chinese Medicine (Guangzhou, China).

To observe the biodistribution of liposomes in vivo, the liposomes were first stained with DiI at $37^{\circ} \mathrm{C}$ for $30 \mathrm{~min}$. MEAR cells suspended in PBS $\left(2 \times 10^{7}\right.$ cells $\left./ \mathrm{ml} ; 0.2 \mathrm{ml}\right)$ was subcutaneously injected into the right lower limb of BALB/c nude mice. The tumor length and width of all mice were monitored every other day following the subcutaneous injection of MEAR cells. The tumor volumes were calculated using the following equation, subsequent to measuring the width and length of each tumor with a calliper:

$$
\text { Tumor volu me }\left(\mathrm{mm}^{3}\right)=\frac{\text { width }^{2} \times \text { length }}{2}
$$

Until the tumor volumes reached $\sim 50 \mathrm{~mm}^{3}, 200 \mu 1$ BioTL-Cab/lipo/DiI or Cab/lipo/DiI ( $n=3$ mice per group) was injected intravenously via the tail vein. At different time points $(1,2,4,6$ and $24 \mathrm{~h})$ post-injection, mice were anesthetized and scanned using an IVIS ${ }^{\circledR}$ Lumina XRMS Series III (PerkinElmer Inc., Waltham, MA, USA) with excitation and emission wavelengths of 549 and $565 \mathrm{~nm}$, respectively.

Antitumor efficacy in mice. Male BALB/c nude mice $(n=36$; 5 weeks-old, $20 \mathrm{~g}$ ) were obtained from the Experimental Animal Center at Guangzhou University of Chinese Medicine (Production license no. 44005800003020). The mice were housed in a temperature-controlled room $\left(22-25^{\circ} \mathrm{C}\right)$, with $50-60 \%$ relative humidity under a $12 \mathrm{~h}$ light-dark cycle with free access to food and water. When the MEAR cell tumor volumes reached $\sim 50 \mathrm{~mm}^{3}$, chemotherapy was performed on the xenograft mice. Mice were weighed and randomly divided in 6 groups ( $n=6$ mice per group): i) Negative control group (NS; $200 \mu 1$ normal saline solution); ii) drug solution group [200 $\mu \mathrm{l} \mathrm{Cab}$ solution $(1 \mathrm{mg} / \mathrm{ml})$, equal to $10 \mathrm{mg} / \mathrm{kg}$ of $\mathrm{Cab}$ to mice]; iii) high BioTL-Cab/lipo dose group [HBG; $300 \mu \mathrm{l}$ BioTL-Cab/lipo solution ( $1 \mathrm{mg} / \mathrm{ml})$, equal to $15 \mathrm{mg} / \mathrm{kg}$ of Cab to mice]; iv) middle BioTL-Cab/lipo dose group [MBG; $200 \mu \mathrm{l} \mathrm{BioTL-Cab/lipo} \mathrm{solution} \mathrm{(} 1 \mathrm{mg} / \mathrm{ml})$, equal to $10 \mathrm{mg} / \mathrm{kg}$ of $\mathrm{Cab}$ to mice]; v) low BioTL-Cab/lipo dose group [LBG; $100 \mu \mathrm{l} \mathrm{BioTL-Cab/lipo} \mathrm{solution}(1 \mathrm{mg} / \mathrm{ml})$, equal to $5 \mathrm{mg} / \mathrm{kg}$ of Cab to mice]; and vi) Cab/lipo group [200 $\mu \mathrm{l} \mathrm{Cab/lipo} \mathrm{solu-}$ tion $(1 \mathrm{mg} / \mathrm{ml})$, equal to $10 \mathrm{mg} / \mathrm{kg}$ of Cab to mice]. The mice received injections every three days via the tail vein for three times in total. The mice were sacrificed $48 \mathrm{~h}$ after the final injection, and the tumors were removed and weighed.

Statistical analysis. The results were presented as the mean \pm standard deviation of three independent experiments. Statistical analysis was performed using SPSS software, version 20 (IBM Corp., Armonk, NY, USA); one-way analysis of variance with a Dunnett's test or a Student's t-test were performed. $\mathrm{P}<0.05$ was considered to indicate a statistically significant difference.

\section{Results}

Physicochemical characterization of liposomes. The in vivo characteristics of liposomes is affected by numerous factors, including particle size and surface charge (14). Table I demonstrates the size, $\zeta$ potential and EE\% of Cab/lipo, BioTL-Cab/lipo, 6-FAM/lipo and BioTL-6-FAM/lipo. The particle size of the liposomes was $80-100 \mathrm{~nm}$ with an acceptable polydispersity index of $0.246-0.273$. The particle size of the liposomes markedly increased in the presence of BioTL in Cab/lipo and 6-FAM/lipo. BioTL increased the EE of Cab by liposomes, but reduced that of 6-FAM by liposomes in the process of emulsification. BioTL is a single-stranded DNA (ssDNA) with a surfactant effect that may increase or reduce 
Table I. Size and EE of liposomes $(n=3)$.

\begin{tabular}{lcccc}
\hline Treatment & Size $(\mathrm{nm})$ & PDI & $\zeta(\mathrm{mV})$ & EE $(\%)$ \\
\hline Cab/lipo & $82.73 \pm 3.82$ & 0.261 & $-50.2 \pm 4.34$ & $93.19 \pm 2.29^{\mathrm{a}}$ \\
BioTL-Cab/lipo & $90.10 \pm 2.71$ & 0.246 & $-58.6 \pm 2.40$ & $97.26 \pm 3.18^{\mathrm{a}}$ \\
6-FAM/lipo & $90.98 \pm 1.21$ & 0.245 & $-45.4 \pm 1.50$ & $89.63 \pm 3.12^{\mathrm{b}}$ \\
BioTL-6-FAM/lipo & $96.98 \pm 1.24$ & 0.273 & $-55.8 \pm 2.04$ & $83.63 \pm 3.05^{\mathrm{b}}$
\end{tabular}

${ }^{\mathrm{a}} \mathrm{EE} \%$ of Cab, ${ }^{\mathrm{b} E} \%$ of 6-FAM. 6-FAM, 6-carboxy fluorescein; BioTL, biotinylated TLS1c; Cab, cabazitaxel; EE, encapsulation efficiency; lipo, liposome; PDI, polydispersity index.

Table II. Effect of BioTL/SPC ratio on size and EE\% of liposomes $(n=3)$.

\begin{tabular}{lccrr}
\hline BioTL ratio to SPC $(\mu \mathrm{g} / \mathrm{mg})$ & Size $(\mathrm{nm})$ & PDI & $\zeta(\mathrm{mV})$ & EE $(\%)$ \\
\hline 0.2 & $85.87 \pm 1.39$ & 0.288 & $-54.3 \pm 2.65$ & $93.17 \pm 3.91$ \\
1 & $90.10 \pm 2.71$ & 0.246 & $-58.6 \pm 2.40$ & $97.26 \pm 3.18$ \\
2 & $92.76 \pm 1.22$ & 0.273 & $-57.4 \pm 1.42$ & $91.64 \pm 3.27$ \\
\hline
\end{tabular}

BioTL, biotinylated TLS1c; EE, encapsulation efficiency; PDI, polydispersity index; SPC, soya phosphatidyl choline.

the EE of drugs by liposomes. Cab/lipo and BioTL-Cab/lipo exhibited an increased EE\% compared with 6-FAM/lipo or BioTL-6-FAM/lipo. The solubility of 6-FAM is lower than that of $\mathrm{Cab}$ in dichloromethane due to the differing chemical properties of Cab and 6-FAM $(15,16)$. Generally, all of the liposome samples had a $\zeta$ potential $<-45 \mathrm{mV}$, demonstrating that the particles were negatively charged, and thus, electrostatic interaction may prevent them from aggregating.

In the present study, based on the nucleic acid properties of the aptamer TLS1c (single-stranded DNA), an Ultra-Micro spectrophotometer was used for the quantification of free or loosely attached BioTL. A ratio of $1 \mu \mathrm{g} / \mathrm{mg}$ BioTL to SPC had been applied to the liposomes and the percentage of free BioTL was $1.09 \pm 0.91 \%$, suggesting that almost all aptamers were captured by liposomes (data not shown).

The EE\% and the size of liposomes with different ratios of BioTL to SPC are presented in Table II. The highest EE\% and absolute $\zeta$ potential value of liposomes were observed with the BioTL to SPC ratio of $1 \mu \mathrm{g} / \mathrm{mg}$. The ratio of $2 \mu \mathrm{g} / \mathrm{mg}$ resulted in the lowest $\mathrm{EE} \%$, which may be due to a large excess of aptamers, preventing encapsulation of the drug.

The results from flow cytometry analysis (Fig. 1), combined with the data presented in Table II, suggested that $1 \mu \mathrm{g} / \mathrm{mg}$ BioTL was the optimal ratio added to the liposomes regarding the binding rate. As presented Fig. 1, a time-dependent increase in the fluorescence intensity of MEAR cells was observed within $5 \mathrm{~h}$ incubation. At $5 \mathrm{~h}$, the liposomes modified with $0.2 \mu \mathrm{g} / \mathrm{mg}$ aptamer exhibited lower binding ability to cells, with a mean fluorescence intensity of 3,066. Application of $1 \mu \mathrm{g} / \mathrm{mg}$ aptamer revealed increased cell binding ability, with a mean fluorescence intensity of 4,761, which was significantly higher compared with $0.2 \mu \mathrm{g} / \mathrm{mg}$ aptamer at $5 \mathrm{~h}(\mathrm{P}<0.01 \mathrm{vs}$. the ratios of $1 \mu \mathrm{g} / \mathrm{mg}$ BioTL/SPC at $5 \mathrm{~h}$ ); however, with $2 \mu \mathrm{g} / \mathrm{mg}$ aptamer, the mean fluorescence intensity reached 4,805 , which

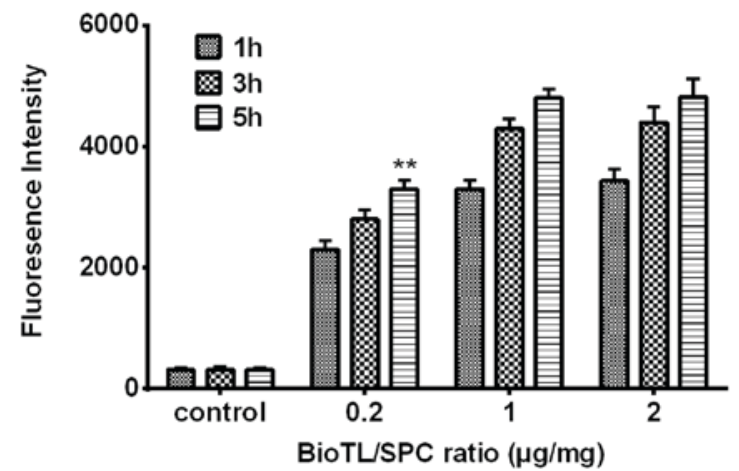

Figure 1. Flow cytometry analysis of fluorescence intensity of MEAR cells treated with different ratios of BioTL (aptamers) to SPC $(0.2,1$ and $2 \mu \mathrm{g} / \mathrm{mg}$ ), measured at incubation times of 1,3 and $5 \mathrm{~h}$ (excitation wavelength, $452 \mathrm{~nm}$, emission wavelength, $521 \mathrm{~nm}$ ). ${ }^{* *} \mathrm{P}<0.01 \mathrm{vs}$. the ratios of $1 \mu \mathrm{g} / \mathrm{mg}$ BioTL/SPC at 5 h. MEAR, BNL 1ME A.7R.1 cells; BioTL, biotinylated TLS1c; SPC, soya phosphatidyl choline.

was markedly higher compared with $1 \mu \mathrm{g} / \mathrm{mg}$. These data suggested that as the ratio exceeded $1 \mu \mathrm{g} / \mathrm{mg}$, the binding of liposomes to MEAR cells may be less affected by aptamer concentration. Considering the EE (\%) results in Table II, $1 \mu \mathrm{g} / \mathrm{mg}$ BioTL was determined as the optimal ratio to modify liposomes.

Cytotoxicity assays. The cytotoxicity of Cab was determined prior to other cell experiments. As presented in Fig. 2A, the cytotoxicity of $\mathrm{Cab}$ on viability demonstrated a significant dose-dependent effect in MEAR cells. Cell viability also decreased in all conditions with increasing incubation time. The inhibitory rate significantly increased in response to various concentrations $(0.0625-2 \mu \mathrm{g} / \mathrm{ml})$ at an incubation time of $72 \mathrm{~h}$ compared to the control group. The half maximal 
A

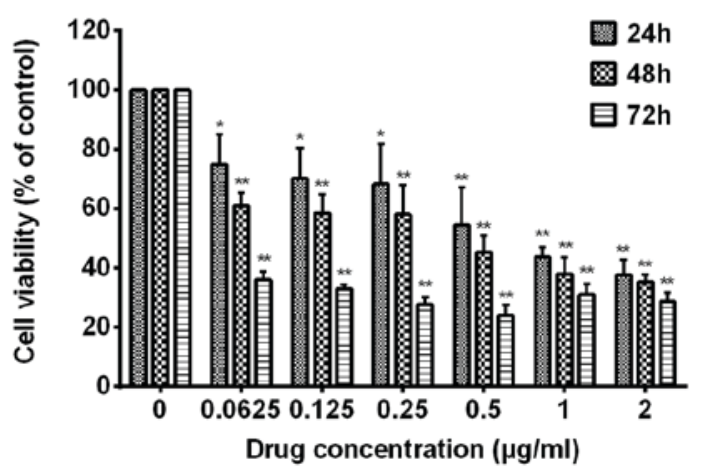

B

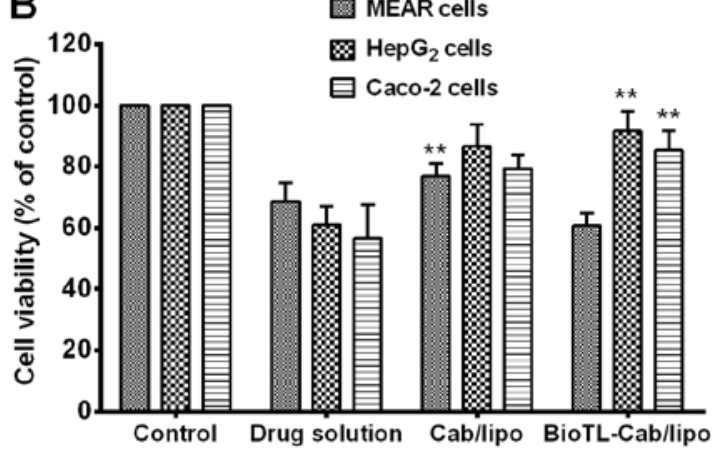

Figure 2. Effect of Cab solution, Cab/lipo and BioTL-Cab/lipo on the inhibition of MEAR, HepG2 and Caco-2 cell viability. (A) Viability of MEAR cells treated with different concentrations of Cab solution after 24,48 and $72 \mathrm{~h}$. ${ }^{*} \mathrm{P}<0.05,{ }^{* *} \mathrm{P}<0.01$ vs. Control. (B) Viability of MEAR, HepG2 and Caco-2 cells treated with Cab solution, Cab/lipo and BioTL-Cab/lipo after $24 \mathrm{~h}$. All values are presented as the mean \pm standard deviation. ${ }^{* *} \mathrm{P}<0.01 \mathrm{vs}$. BioTL-Cab/lipo-treated MEAR cells, Cab/lipo or drug solution. MEAR, BNL 1ME A.7R.1 cells; Cab, cabazitaxel; BioTL, biotinylated TLS1c; lipo, liposomes.

inhibitory concentrations of $\mathrm{Cab}$ to MEAR were determined to be $0.65,0.48$ and $0.21 \mu \mathrm{g} / \mathrm{ml}$ for 24,48 and $72 \mathrm{~h}$ of treatment, respectively.

As presented in Fig. 2B, cell viability notably increased in response to $0.5 \mu \mathrm{g} / \mathrm{ml} \mathrm{Cab} /$ lipo compared with the drug solution in all the three cell types, which suggested that liposomes may decrease the cytotoxicity of the drug directly. Furthermore, it was identified that the viability of Caco-2 or HepG2 cells incubated with BioTL-Cab/lipo was significantly increased compared with the drug solution and Cab/lipo; however, the highest levels of cytotoxicity were observed in MEAR cells when treated with BioTL-Cab/lipo compared with Cab/lipo. Based on the results of the cell cytotoxicity assays, it was speculated that the modification of BioTL to Cab/lipo may improve specificity for MEAR cells.

Cellular uptake studies. In the preliminary experiments, the cellular uptake of liposomes in MEAR, Caco-2 and HepG2 cells was determined by CLSM (Fig. 3). The fluorescence intensity observed in 6-FAM/lipo-treated MEAR cells was notably lower compared with BioTL-6-FAM/lipo; the fluorescence intensity of 6-FAM/lipo-treated Caco-2 cells was a notably higher compared with BioTL-6-FAM/lipo, which demonstrated that 6-FAM/lipo was engulfed by Caco-2 cells at a higher degree compared with BioTL-6-FAM/lipo. A similar phenomenon for 6-FAM/lipo-treated HepG2 cells was observed as the fluorescence intensity was markedly higher compared with BioTL-6-FAM/lipo-treated HepG2 cells, was detected. The images supported the observations of the present study that BioTL-6-FAM/lipo exhibits higher specificity for MEAR cells; the green fluorescence signals of BioTL-6-FAM/lipo-treated Caco-2 cells or HepG2 cells were weaker compared with BioTL-6-FAM/lipo-treated MEAR cells. These results demonstrated the targeting effect of BioTL-6-FAM/lipo to MEAR cells in vitro. The accumulation of green fluorescence signals was mainly detected in the cytoplasmic area of all the cells.

Flow cytometry analysis was performed to quantitatively measure the cellular uptake of liposomes. MEAR cells were incubated overnight, and 6-FAM/lipo or BioTL-6-FAM/lipo was applied for an additional $3 \mathrm{~h}$. The quantitative flow cytometry analysis demonstrated that the cellular uptake of 6-FAM/lipo-treated MEAR cells was notably decreased compared with MEAR cells treated with BioTL-6-FAM/lipo (Fig. 4A), possibly due to the targeting specificity of BioTL to MEAR cells. Additionally, a significant increase in fluorescence intensity was observed in BioTL-6-FAM/lipo-treated MEAR cells compared with 6-FAM/lipo-treated MEAR cells (Fig. 4B). This demonstrated the high specificity of BioTL-6-FAM/lipo to MEAR cells compared with Caco-2, HepG2 cells or macrophages. These results suggested the selectivity of BioTL-liposomes for MEAR cells compared with the Caco-2 and HepG2 cells, which is in accordance with the findings of the cytotoxicity assay (Fig. 2B). Based on the aforementioned findings, it may be concluded that the aptamer (BioTL)-modified liposomes exhibited high specificity to the target cells. Although the cellular internalization process of the TLS1c aptamer has not been clearly elucidated, these results demonstrated that TLS1c was able to induce the efficient delivery of liposomes to MEAR cells. The results of the CLSM and flow cytometry assay demonstrated the specific targeting of BioTL-Cab/lipo to MEAR cells.

In vivo biodistribution of BioTL-Cab/lipo. High affinity of liposomes to tumor cells is a critical factor contributing to high antitumor efficacy in vivo. The use of fluorescence imaging to monitor local tumor accumulation and functional activity of magnetic nanoparticles covalently linked to small interfering RNAs was previously described (17). The real-time biodistribution of liposomes in tumor-bearing nude mice was evaluated with in vivo images in the present study. The tumor-bearing mice were used for the observation of the biodistribution of BioTL liposomes stained with DiI. In total, $200 \mu 1$ BioTL-Cab/lipo/DiI or Cab/lipo/DiI were intravenously injected into the mice. As presented in Fig. 5, strong signals were observed in the blood-rich tissues, including the brain, heart and lungs. As Cab/lipo/DiI was intravenously injected in the nude mice, the fluorescence signal at the tumor location was almost undetectable. Conversely, the fluorescence signals at the tumor locations in the BioTL-Cab/lipo/DiI-treated mice gradually increased with time ( $\leq 24 \mathrm{~h}$ ). Cab/lipo/DiI primarily circulated 


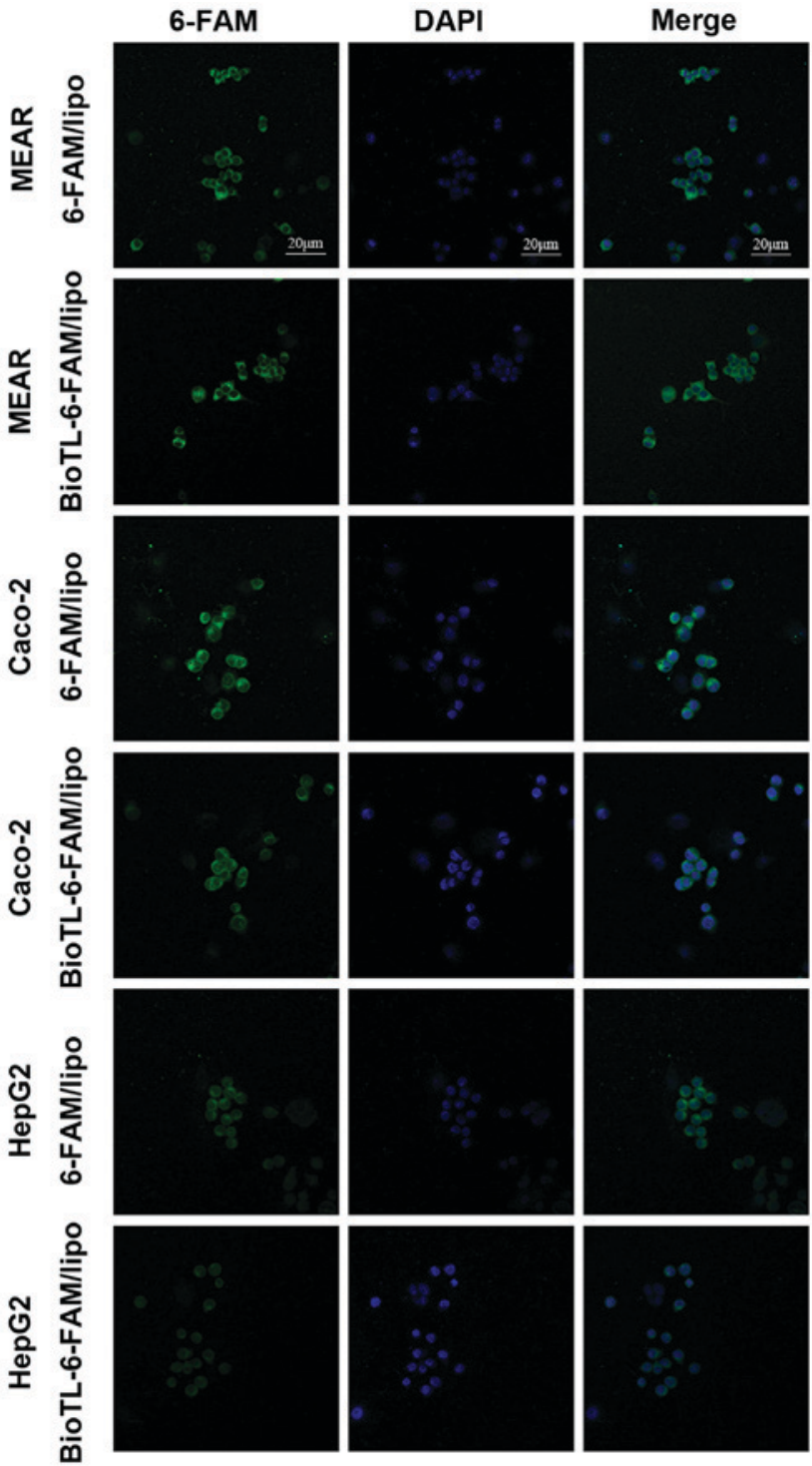

Figure 3. Confocal laser scanning microscopy images of DAPI (blue) and 6-FAM (green) of MEAR, Caco-2 or HepG2 cells treated with 6-FAM/Lipo or BioTL-6-FAM/Lipo for $3 \mathrm{~h}$. MEAR, BNL 1ME A.7R.1 cells; BioTL, biotinylated TLS1c; 6-FAM, 6-carboxy fluorescein; lipo, liposomes.

in the bloodstream, but did not enter the tumor tissue (Fig. 5). On the contrary, the liposomal system of BioTL-Cab/lipo/DiI accumulated in tumor tissue. Furthermore, time-dependent fluorescence signals were identified for BioTL-Cab/lipo/DiI, suggesting that the liposomes modified with BioTL gradually accumulated in tumor tissue as a function of time. These results suggested that the BioTL-modified liposomes may deliver antitumor drugs directly to the tumor with long-term retention, which is critical for enhancing any antitumor effect.

In vivo antitumor efficacy. To investigate the in vivo therapeutic efficacy of BioTL-Cab/lipo, an antitumor study was performed on subcutaneously inoculated MEAR tumor-bearing mouse models. The tumor volume in MEAR tumor-bearing mice as a function of time (days) during the study was presented in Fig. 6A. The NS group demonstrated a rapid increase in tumor volume over the whole experimental period. Conversely, the HBG exhibited the most notable tumor suppression, in which the tumor volume decreased from $50 \mathrm{~mm}^{3}$ on day 5 to $\sim 28 \mathrm{~mm}^{3}$ on day 13, which demonstrated that the tumor volume was significantly decreased compared to the NS group.

For tumor weight analysis, mice were sacrificed $48 \mathrm{~h}$ after the last administration. The tumor tissue was removed and weighed (Fig. 6B), and alterations in the body weight were associated with the time of treatment (Table III). Alterations in standardized body weight of mice administered the drug solution and $\mathrm{Cab} /$ lipo were $<0 \mathrm{~g}$, suggesting that the side effects of $\mathrm{Cab}$ were harmful to mice; however, the changes in standardized body weight of the three groups of mice administered BioTL-Cab/lipo were $>0 \mathrm{~g}$, indicating that the toxicity of $\mathrm{Cab}$ decreased following modification with the same amount of aptamer.

The results of the present study suggested that BioTL-Cab/lipo exerted considerably enhanced inhibitory effects on tumor growth compared with Cab/lipo or the drug solution in the MEAR cell-induced tumor xenografts. According to these results, antitumor drugs, particularly with similar physical/chemical properties to Cab, may also be successfully delivered by the developed BioTL/lipo system with similar targeting effects. Therefore, the results of the present study may provide insight into the development of BioTL/lipo-based approaches for a variety of anticancer treatments.

\section{Discussion}

The present study demonstrated that aptamer (TLS1c)-modified Cab liposomes decreased Cab cytotoxicity in mice. Compared with proteins or peptides, oligonucleotide aptamers are easier to chemically prepare and modify, and scale up in production (18-21). Furthermore, aptamers are generally nonimmunogenic $(22,23)$ and may be rapidly removed from the blood with minimal system toxicity (24). These advantages suggest aptamers as alternative targeting elements for preparing 'immuno-liposomes'. Therefore, the present study aimed to develop a targeting system for drug delivery with aptamers. As of the advantages of liposomes, including high efficiency to encapsulate hydrophilic drugs, protection of the encapsulated drugs from external conditions, sustained-release of drugs, ease of surface modification, and most importantly, the ability of decreasing drug toxicity, they have been employed as drug delivery vectors in previous aptamer-targeting studies (25). Li et al (26) successfully developed a method of selective delivery using liposomes for the treatment of malignant melanomas with AS1411, a DNA aptamer with strong binding affinity for nucleolin, which demonstrated the potential of aptamer-liposome conjugates in the application of targeting treatment; however, few studies using aptamer-conjugated Cab liposomes for cancer cell targeting have been conducted. The aim of the present study was to develop a novel liposomal delivery system based on the specificity of the aptamer TLS1c for MEAR cells to decrease the toxicity of $\mathrm{Cab}$ in vitro and in vivo.

When BioTL is modified on the surface of liposomes, it may form a film, which improves the specificity of liposomes for target cells (27). Although, when the ratio was $>1 \mu \mathrm{g} / \mathrm{mg}$ aptamer to SPC, the abundance of aptamers markedly increased; however, a significant increase in fluorescence intensity was not observed. This may be explained by the limitation of targets on cells. As the ratio of aptamer to SPC was $1 \mu \mathrm{g} / \mathrm{mg}$, 
A

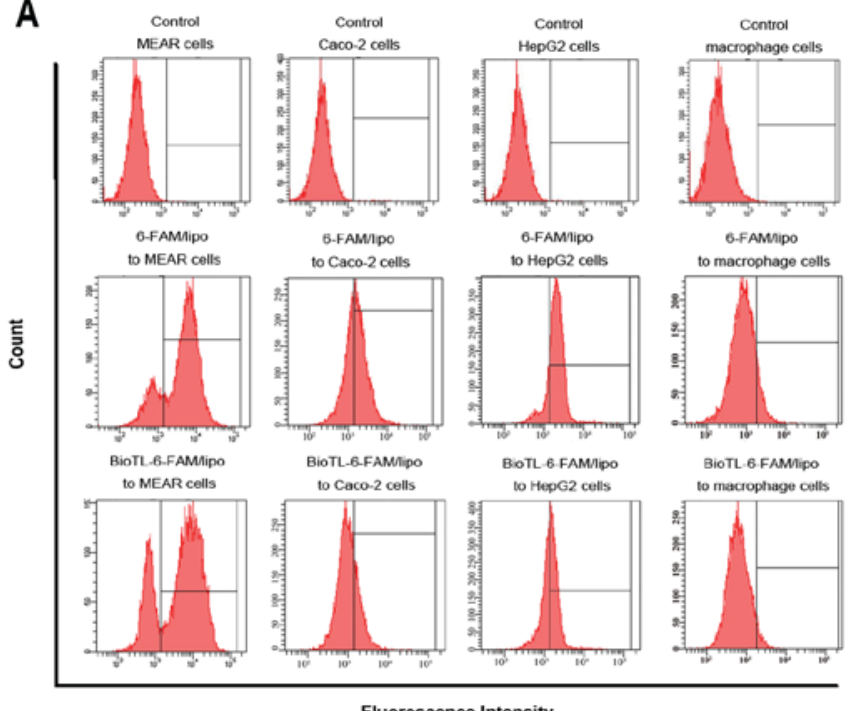

B

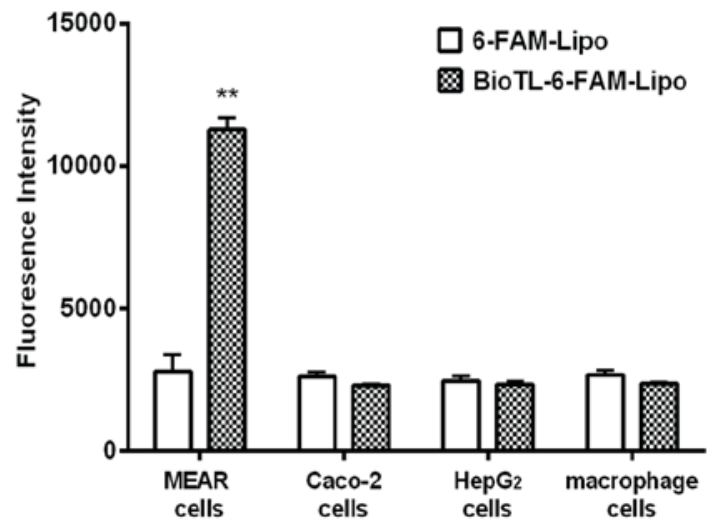

Figure 4. Effect of MEAR, Caco-2, HepG2 or macrophage cells on the uptake of 6-FAM/Lipo or BioTL-6-FAM/Lipo. (A) Quantitative flow cytometry analysis of cells following treatment for $3 \mathrm{~h}$. (B) Flow cytometry analysis of fluorescence intensity. All values are presented as the mean \pm standard deviation. ${ }^{* * *} \mathrm{P}<0.01$ vs. 6-FAM-Lipo-treated MEAR cells. MEAR, BNL 1ME A.7R.1 cells; 6-FAM, 6-carboxy fluorescein; FITC, fluorescein isothiocyanate; lipo, liposomes.

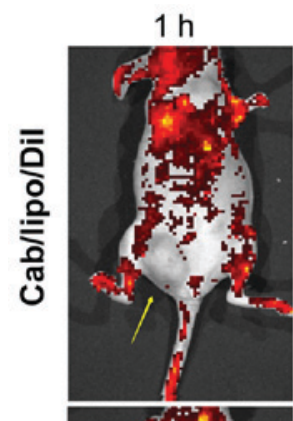

$2 \mathrm{~h}$
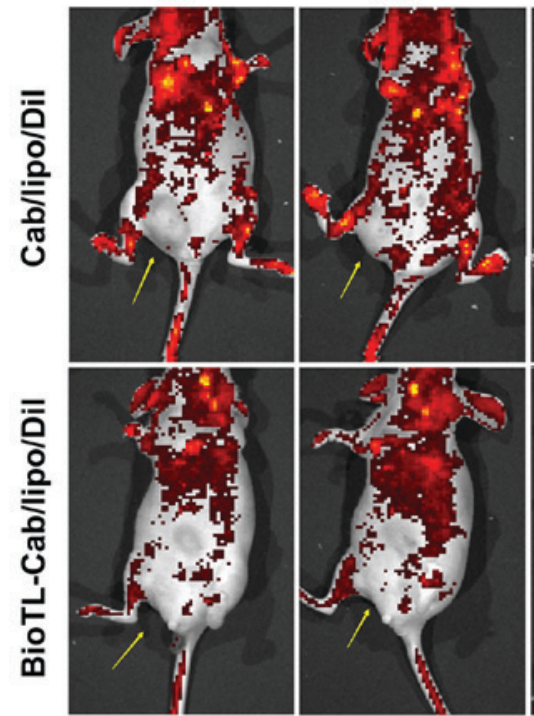

$4 \mathrm{~h}$
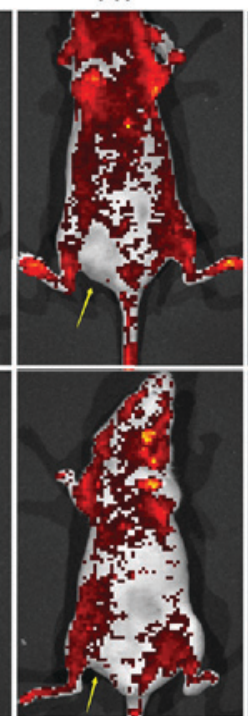

$6 \mathrm{~h}$

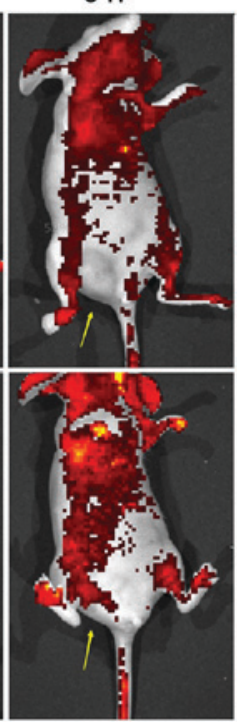

$24 \mathrm{~h}$

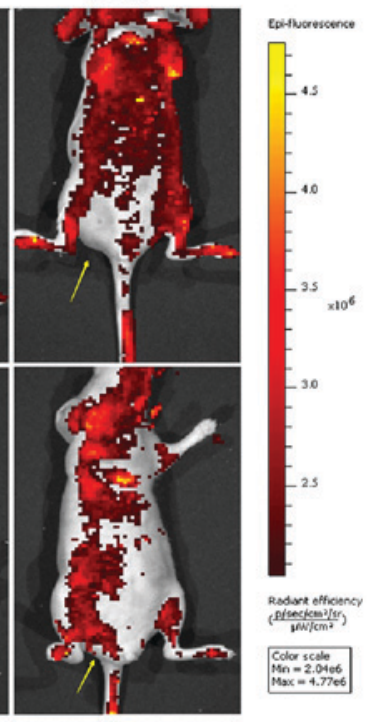

Figure 5. In vivo real-time images of mice following tail vein injection of liposomes at different times for $24 \mathrm{~h}$. The yellow arrow indicates the location of the tumor. BioTL, biotinylated TLS1c; Cab, cabazitaxel; Dil, 1,1'-dioctadecyl-3,3,3',3'-tetramethylindocarbocyanine perchlorate.

aptamers may combine well with the target. Conversely, upon application of $2 \mu \mathrm{g} / \mathrm{mg}$ aptamer to SPC, no notable alterations in targeting ability were reported. Therefore, the modification ratio of BioTL to SPC was selected as $1 \mu \mathrm{g} / \mathrm{mg}$.

Investigation into the effects of BioTL-Cab/lipo on cell viability revealed increased cell viability of the three cell lines compared with the drug solution. Additionally, these results suggested that $\mathrm{Cab} /$ lipo may not possess the specificity to target cells. Conversely, the highest cytotoxic effects of BioTL-Cab/lipo were observed in MEAR cells. These observations suggested that the targeting ability of Cab/lipo improved following conjugation with BioTL. The results of CLSM and flow cytometry analyses demonstrated that BioTL-Cab/lipo specifically targeted MEAR cells. Liposomes are endocytosed by cells and expel their contents into the cytoplasm $(28,29)$. In the present study, the fluorescence signal of 6-FAM was also detected in the cytoplasm. The accumulation of liposomes with or without aptamers was observed in the cytoplasm; however, the mechanism underlying the internalization of BioTL-Cab/lipo into cells remains unclear. Further investigation into the mechanism of BioTL-Cab/lipo internalization by MEAR cells may be conducted in the future.

The therapeutic efficacy of drug-loaded nanoparticles is affected by the accumulation of drugs in tumor cells in vivo or in vitro; accumulation depends on the cellular uptake and distribution characteristics of the nanoparticles (30). In the present study, analysis of the biodistribution and antitumor efficacy of BioTL-Cab/lipo in vivo demonstrated the targeting 
Table III. Alterations in body weight following treatment $(n=3)$.

\begin{tabular}{lcccccc}
\hline & \multicolumn{7}{c}{ Body weight $(\mathrm{g})$} \\
\cline { 2 - 7 } Point of analysis & NS & Cab/lipo & HBG & MGB & LBG & Drug solution \\
\hline Pre-administration & $20.13 \pm 1.18$ & $19.72 \pm 3.35$ & $18.32 \pm 3.14$ & $16.57 \pm 2.24$ & $17.21 \pm 1.14$ & $20.30 \pm 3.27$ \\
Post-administration & $23.7 \pm 1.70$ & $20.17 \pm 3.08$ & $19.26 \pm 1.96$ & $17.88 \pm 1.68$ & $20.97 \pm 1.67$ & $18.00 \pm 1.17$ \\
Difference & 3.57 & 0.45 & 0.94 & 1.31 & 3.76 & -2.30 \\
\hline
\end{tabular}

Cab/lipo, liposome containing cabazitaxel; HBG, high BioTL-Cab/lipo dose group; LBG, low BioTL-Cab/lipo dose group; MBG, middle BioTL-Cab/lipo dose group; NS, negative control.

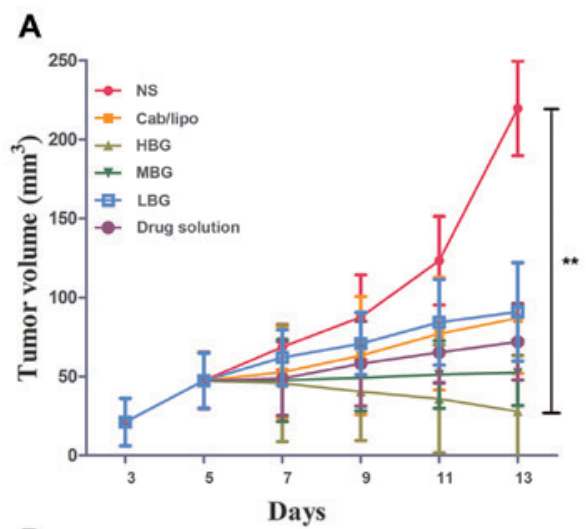

B

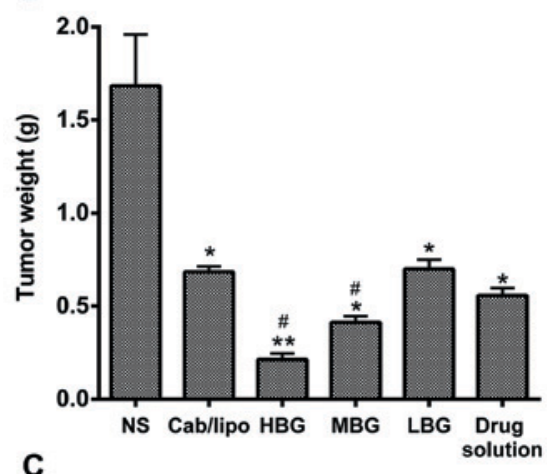

C

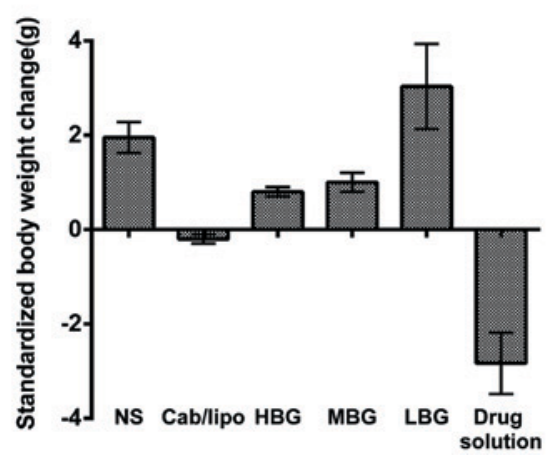

Figure 6. Antitumor effect on MEAR-tumor bearing mice treated with liposomes and drug solution compared with the NS group. (A) Tumor volume alters as a function of time following intravenous injection at $48 \mathrm{~h}$ after the last injection. ${ }^{* *} \mathrm{P}<0.01$ vs. the NS group. (B) Tumor weight of the mice treated at $48 \mathrm{~h}$ after the last injection. (C) Standardized body weight of mice treated at $48 \mathrm{~h}$ after the last injection. $\mathrm{n}=6$. All values are presented as the mean \pm standard deviation. ${ }^{*} \mathrm{P}<0.05,{ }^{* *} \mathrm{P}<0.01$ vs. the NS group. ${ }^{~} \mathrm{P}<0.05$ vs. the $\mathrm{Cab} / \mathrm{lipo}$. Cab/lipo, cabazitaxel liposomes; HBG, high BioTL-Cab/lipo dose group; LBG, low BioTL-Cab/lipo dose group; MBG, middle BioTL-Cab/lipo dose group; MEAR, BNL 1ME A.7R.1; NS, negative control. of MEAR cells. The specific targeting of BioTL-Cab/lipo to the tumor location was also observed; however, accumulation in the brain, heart or lungs was reported, which may be due to the intrinsic high blood perfusion rate of the circulatory system. Furthermore, the tumor volume significantly decreased in response to a high dose of BioTL-Cab/lipo. The findings of the present study supported the initial hypothesis that the cytotoxicity of $\mathrm{Cab}$ decreased with aptamer-modified liposomes.

Accumulating evidence has suggested that BioTL, an aptamer with a short DNA sequence, specifically targeted MEAR hepatoma cells $(10,12)$; however, the effects of BioTL-Cab/lipo on MEAR, Caco-2, macrophages and HepG2 cells remain unknown. Therefore, the present study aimed to investigate whether BioTL-Cab/lipo could specifically target MEAR cells, and not Caco-2, macrophages and HepG2 cells. To the best of our knowledge, the present study is the first to demonstrate the development of liposomes co-modified with $\mathrm{Cab}$ and the aptamer TLS1c to target MEAR cells. The results suggested that TLS1c-conjugated liposomes successfully targeted MEAR cells. In addition, the complexes of BioTL-Cab/lipo demonstrated significant inhibition of the viability of MEAR cells in vitro and in vivo. It was proposed that the aptamer-modified liposome delivery system may be an effective and safe strategy for the delivery of Cab to MEAR cells.

In conclusion, a novel liposome delivery system based on the specificity of aptamer TLS1c for MEAR cells was developed to deliver Cab to MEAR cells. BioTL-Cab/lipo was designed as a targeting drug delivery system to decrease the toxicity of Cab. As a result, BioTL-Cab/lipo was associated with increased cellular uptake and reduced cytotoxicity in MEAR cells compared with non-modified liposomes (Cab/lipo). Furthermore, BioTL-Cab/lipo notably accumulated at the tumor site. Significant decrease in tumor volume and weight were observed when BioTL-Cab/lipo was intravenously administered; systemic side effects, such as animal weight loss in the model inoculated with MEAR cells, were decreased compared with the drug solution. These results suggested that BioTL-Cab/lipo may be a promising system for targeting tumor cells and decreasing drug toxicity.

\section{Acknowledgements}

Not applicable. 


\section{Funding}

The present study was supported by the Guangzhou Science and Technology Project (grant no. 201604020166; China).

\section{Availability of data and materials}

The datasets used during the present study are available from the corresponding author upon reasonable request.

\section{Authors' contributions}

$\mathrm{YC}$ and $\mathrm{ZO}$ designed and conceived this study. $\mathrm{YC}$ and $\mathrm{ZO}$ performed the experiments. QL, YZ and XZ performed the animal experiments and cell culture. JY, ZJZ and MH conducted statistical analysis of the results. YC and ZO wrote the paper. SG reviewed and edited the manuscript, acquired funds and contributed to the study design. All authors read and approved the manuscript, and agree to be accountable for all aspects of the research in ensuring that the accuracy or integrity of any part of the work are appropriately investigated and resolved.

\section{Ethics approval and consent to participate}

The present study was approved by the Animal Research Ethics Committee at Guangzhou University of Chinese Medicine (Guangzhou, China). All animal treatments were performed in accordance with the Regulations of the Administration of Affairs Concerning Experimental Animals.

\section{Patient consent for publication}

Not applicable.

\section{Competing interests}

The authors declare that they have no competing interests.

\section{References}

1. Force $T$ and Kerkela R: Cardiotoxicity of the new cancer therapeutics-mechanisms of, and approaches to, the problem. Drug Discov Today 13: 778-784, 2008.

2. Tuerk C and Gold L: Systematic evolution of ligands by exponential enrichment: RNA ligands to bacteriophage t4 DNA polymerase. Science 249: 505-510, 1990.

3. Shangguan D, Li Y, Tang Z, Cao ZC, Chen HW, Mallikaratchy P, Sefah K, Yang CJ and Tan W: Aptamers evolved from live cells as effective molecular probes for cancer study. Proc Natl Acad Sci USA 103: 11838-11843, 2006.

4. Taghdisi SM, Lavaee P, Ramezani M and Abnous K: Reversible Targeting and controlled release delivery of daunorubicin to cancer cells by aptamer-wrapped carbon nanotubes. Eur J Pharm Biopharm 77: 200-206, 2011.

5. Shigdar S, Macdonald J, O'Connor M, Wang T, Xiang D, Al Shamaileh H, Qiao L, Wei M, Zhou SF, Zhu Y, et al: Aptamers as theranostic agents: Modifications, serum stability and functionalisation. Sensors (Basel) 13: 13624-13637, 2013.

6. Fang X and Tan W: Aptamers generated from cell-SELEX for molecular medicine: A chemical biology approach. Acc Chem Res 43: 48-57, 2010.

7. Alibolandi M, Ramezani M, Abnous K and Hadizadeh F: AS1411 aptamer-decorated biodegradable polyethylene glycol-poly(lactic-co-glycolic acid) nanopolymersomes for the targeted delivery of gemcitabine to non-small cell lung cancer in vitro. J Pharm Sci 105: 1741-1750, 2016.
8. Keefe AD, Pai S and Ellington A: Aptamers as therapeutics. Nat Rev Drug Discov 9: 537-550, 2010.

9. Sperlich C and Saad F: Optimal management of patients receiving cabazitaxel-based chemotherapy. Can Urol Assoc J 7 (1-2 Suppl 1): S18-S24, 2013.

10. Shangguan D, Meng L, Cao ZC, Xiao Z, Fang X, Li Y, Cardona D, Witek RP, Liu C and Tan W: Identification of liver cancer-specific aptamers using whole live cells. Anal Chem 80: 721-728, 2008.

11. Ishii $\mathrm{M}$, Koyama $\mathrm{A}$, Iseki $\mathrm{H}$, Narumi $\mathrm{H}$, Yokoyama $\mathrm{N}$ and Kojima N: Anti-allergic potential of oligomannose-coated liposome-entrapped Cry $\mathrm{j}$ I as immunotherapy for Japanese cesar pollinosis in mice. Int Immunopharmacol 10: 1041-1046, 2010.

12. Banghan AD and Horne RW: Negative staining of phospholipids and their structural modification by-surface active agents as observed in electron microscope. J Mol Biol 8: 660-668, 1964.

13. Szoka F Jr and Papahadjopoulos D: Procedure for preparation of liposomes with large internal aqueous space and high capture by reverse-phase evaporation. Proc Natl Acad Sci USA 75: 4194-4198, 1978.

14. He C, Hu Y, Yin L, Tang C and Yin C: Effects of particle size and surface charge on cellular uptake and biodistribution of polymeric nanoparticles. Biomaterials 31: 3657-3666, 2010.

15. Sethna SM and Shah NM: The chemistry of coumarins. Chem Rev 36: 1-62, 1945.

16. Malhotra M, Dhingra R, Sharma T, Deep A, Narasimhan B, Phogat $P$ and Sharma PC: Cabazitaxel: A novel drug for hormone-refractory prostate cancer. Mini Rev Med Chem 13: 915-920, 2013.

17. Medarova Z, Pham W, Farrar C, Petkova V and Moore A: In vivo imaging of siRNA delivery and silencing in tumors. Nat Med 13: 372-377, 2007.

18. Chen Z, Tai Z, Gu F, Hu C, Zhu Q and Gao S: Aptamer-mediated delivery of docetaxel to prostate cancer through polymeric nanoparticles for enhancement of antitumor efficacy. Eur J Pharm Biopharm 107: 130-141, 2016.

19. Brown DM and Todd AR: 13. Nucleotides. Part X. Some observations on the structure and chemical behaviour of the nucleic acids. J Chem Soc 52-58, 1952.

20. Beaucage SL and Caruthers MH: Deoxynucleoside phosphoramidites-A new class of key intermediates for deoxypolynucleotide synthesis. Tetrahedron Lett 22: 1859-1862, 1981.

21. Reese CB: Oligo-and poly-nucleotides: 50 years of chemical synthesis. Org Biomol Chem 3: 3851-3868, 2005.

22. Peer D, Karp JM, Hong S, Farokhzad OC, Margalit R and Langer R: Nanocarriers as an emerging platform for cancer therap. Nat Nanotechnol 2: 751-760, 2007.

23. Shrivastava G, Hyodo M, Yoshimura SH, Akita $H$ and Harashima H: Identification of a nucleoporin358-specific RNA aptamer for use as a nucleus-targeting liposomal delivery system. Nucleic Acid Ther 26: 286-298, 2016.

24. Scaggiantie B, Dapas B, Farra R, Grassi M, Pozzato G, Giansante C, Fiotti N, Tamai E, Tonon F and Grassi G: Aptamers as targeting delivery devices or anti-cancer drugs for fighting tumors. Curr Drug Metab 14: 565-582, 2013.

25. Aravind A, Jeyamohan P, Nair R, Veeranarayanan S, Nagaoka Y, Yoshida Y, Maekawa T and Kumar DS: AS1411 aptamer tagged PLGA-lecithin-PEG nanoparticles for tumor cell targeting and drug delivery. Biotechnol Bioeng 109: 2920-2931, 2012.

26. Li L, Hou J, Liu X, Guo Y, Wu Y, Zhang L and Yang Z: Nucleolin-targeting liposomes guided by aptamer AS1411 for the delivery of siRNA for the treatment of malignant melanoma. Biomaterials 35: 3840-3850, 2014.

27. Chu C, Xu P, Zhao H, Chen Q, Chen D, Hu H, Zhao X and Qiao M: Effect of surface ligand density on cytotoxicity and pharmacokinetic profile of docetaxel loaded liposomes. Asian J Pharm Sci 11: 655-661, 2016.

28. Batzei S and Korn ED: Interaction of phospholipid vesicles with cells. Endocytosis and fusion as alternate mechanisms for the uptake of lipid-soluble and water-soluble molecules. J Cell Biol 66: 621-634, 1975.

29. Szymanowskia F, Hugoa AA, Alvesc P, Simõesc PN, Gómez-Zavagliaa A and Péreza PF: Endocytosis and intracellular traffic of cholesterol-PDMAEMA liposome complexes in human epithelial-like cells. Colloids Surf B Biointerfaces 156: 38-43, 2017.

30. Le UM and Cui Z: Biodistribution and tumor-accumulation of gadolinium $(\mathrm{Gd})$ encapsulated in long-circulating liposomes in tumor-bearing mice for potential neutron capture therapy. Int J Pharm 320: 96-103, 2006.

This work is licensed under a Creative Commons Attribution-NonCommercial-NoDerivatives 4.0 International (CC BY-NC-ND 4.0) License. 